\title{
CONSTRAINTS ON AN OPTICAL AFTERGLOW AND ON SUPERNOVA LIGHT FOLLOWING THE SHORT BURST GRB $050813^{1}$
}

\author{
P. Ferrero, ${ }^{2}$ S. F. Sanchez, ${ }^{3}$ D. A. Kann, ${ }^{2}$ S. Klose, ${ }^{2}$ J. Greiner, ${ }^{4}$ J. Gorosabel, ${ }^{5}$ D. H. Hartmann, ${ }^{6}$ \\ A. A. Henden, ${ }^{7}$ P. Møller, ${ }^{8}$ E. Palazzi, ${ }^{9}$ A. Rau ${ }_{10}^{10}$ B. Stecklum, ${ }^{2}$ A. J. Castro-Tirado, ${ }^{5}$ \\ J. P. U. Fynbo, ${ }^{11}$ J. Huorth, ${ }^{11}$ P. Jakobsson, ${ }^{12}$ C. Kouveliotou, ${ }^{13}$ N. Masetti, ${ }^{9}$ \\ E. Pian, ${ }^{14}$ N. R. TANVIR, ${ }^{15}$ AND R. A. M. J. WiJers ${ }^{16}$ \\ Received 2006 October 9; accepted 2007 August 25
}

\begin{abstract}
We report early follow-up observations of the error box of the short burst GRB 050813 using the telescopes at Calar Alto and Observatorio Sierra Nevada, followed by deep VLT FORS2 I-band observations obtained under very good seeing conditions 5.7 and 11.7 days after the event. Neither a fading afterglow nor a rising SN component was found, so the potential GRB host galaxy has not been identified based on a comparison of the two VLT images taken at different epochs. We discuss whether any of the galaxies present in the original 10" XRT error circle could be the host. In any case, the optical afterglow of GRB 050813 was of very low luminosity. We conclude that all these properties are consistent with the binary compact merger hypothesis for the progenitor of GRB 050813.
\end{abstract}

Key words: gamma rays: observations — supernovae: general

\section{INTRODUCTION}

\subsection{Short Bursts}

Much progress is currently being made toward understanding the nature of the progenitors responsible for the class of shortduration, hard gamma-ray bursts (Kouveliotou et al. 1993; see also the Appendix). While the physical link between long-duration, soft gamma-ray bursts and the core collapse of massive stars (e.g., Paczyński 1998) has been conclusively confirmed by the spectroscopic detection of supernova (SN) light following some bursts

\footnotetext{
${ }^{1}$ Based on observations collected at the European Southern Observatory, La Silla, and Paranal, Chile (ESO Program 075.D-0415), and on observations taken at the German-Spanish Calar Alto Observatory and at IAA's Observatorio de Sierra Nevada in Spain.

2 Thüringer Landessternwarte Tautenburg, Sternwarte 5, D-07778 Tautenburg, Germany.

${ }^{3}$ Centro Astronómico Hispano Alemán de Calar Alto, Calle Jesus Durban Remon 2-2, E-04004 Almería, Spain.

${ }^{4}$ Max-Planck-Institut für Extraterrestische Physik, Giessenbachstrasse, D-85741 Garching, Germany.

${ }^{5}$ Instituto de Astrofísica de Andalucía (IAA-CSIC), Apartado de Correos 3.004, E-18080 Granada, Spain.

${ }^{6}$ Department of Physics and Astronomy, Clemson University, Clemson, SC 29634-0978, USA.

${ }^{7}$ US Naval Observatory/Universities Space Research Association, Flagstaff Station, Flagstaff, AZ 86001, USA.

${ }^{8}$ European Southern Observatory, Karl-Schwarzschild-Strasse 2, D-85748 Garching bei München, Germany.

${ }^{9}$ INAF-Istituto di Astrofisica Spaziale e Fisica Cosmica di Bologna, via Gobetti 101, I-40129 Bologna, Italy.

${ }^{10}$ Division of Physics, Mathematics, and Astronomy, California Institute of Technology, 105-24 Pasadena, CA 91125, USA.

${ }^{11}$ Dark Cosmology Centre, Niels Bohr Insitute, University of Copenhagen, Juliane Maries Vej 30, 2100 Copenhagen, Denmark.

${ }^{12}$ Centre for Astrophysics Research, University of Hertfordshire, College Lane, Hatfield, Herts AL10 9AB, UK.

${ }^{13}$ National Space Science and Technology Center, SD-50, 320 Sparkman Drive, Huntsville, AL 35805, USA.

${ }^{14}$ INAF-Osservatorio Astronomico di Trieste, Via Tiepolo 11, I-34143 Trieste, Italy.

${ }^{15}$ Department of Physics and Astronomy, University of Leicester, Leicester LE1 7RH, UK.

${ }^{16}$ University of Amsterdam, Kruislaan 403, 1098 SJ Amsterdam, The Netherlands.
}

(Stanek et al. 2003; Hjorth et al. 2003; Pian et al. 2006; Woosley $\&$ Bloom 2006 for a review), the nature of the sources responsible for short bursts remains to be revealed in full. Although there is a developing consensus in the community that at least some short bursts are due to merging compact stellar objects (cf. Fryer et al. 1999; Aloy et al. 2005; Rosswog 2005; Oechslin \& Janka 2006; Faber et al. 2006), an unambiguous observational verification of this model is not an easy task and has not yet been accomplished. Furthermore, the origin of a certain fraction of short bursts as giant flares of magnetars in nearby galaxies seems to be possible as well (cf. Tanvir et al. 2005). Indeed, the short hard burst 051103 detected by the Interplanetary Network (Golenetskii et al. 2005) might be the first well-localized member of this class (Frederiks et al. 2007; Ofek et al. 2006).

Within the context of the merger model, the stellar populations underlying short bursts could be associated either with an old stellar population or even with a young one (Belczynski et al. 2006). Short bursts might therefore occur in quiescent ellipticals or star-forming galaxies. Indeed, the first short burst well localized by Swift, GRB 050509B (Gehrels et al. 2005), was associated with a giant elliptical galaxy located in a cluster of galaxies at $z=0.225$ (Bloom et al. 2006; Pedersen et al. 2005), while the HETE-2 short burst GRB 050709 (Hjorth et al. 2005b) occurred in an isolated, star-forming dwarf galaxy. Shortly thereafter, GRB 050724 was found in association with a lone early-type galaxy (Bloom et al. 2005; Prochaska et al. 2005; Berger et al. 2005; Gorosabel et al. 2006). Assuming as a working definition that a short burst should have $T_{90}<2 \mathrm{~s}$, since GRB 050813 six further short bursts were accurately localized by HETE-2 or Swift via their X-ray afterglows by the end of 2006 September (see also Table 8 in Donaghy et al. 2006). Among them GRB 051210 (La Parola et al. 2006), GRB 060502B (Bloom et al. 2007), and GRB 060801 (Racusin et al. 2006) had only X-ray afterglows, while GRB 051221A (Soderberg et al. 2006), GRB 060121 (Malesani et al. 2006; Levan et al. 2006; de Ugarte Postigo et al. 2006), and GRB 060313 (Roming et al. 2006; J. Hjorth et al. 2008 , in preparation) had detected optical afterglows as well. A broad range of morphological types of host galaxies was derived for this set. For example, Bloom et al. (2007) postulated an association between GRB 060502B and a bright elliptical galaxy at 
a large offset at $z=0.287$, while GRB $051221 \mathrm{~A}$ is associated with an isolated star-forming dwarf galaxy (Soderberg et al. 2006), and the host of GRB 060121 might be a dusty edge-on irregular or spiral galaxy (Levan et al. 2006). This "mixed-bag" of host types is consistent with the idea that merging compact binaries will sample all types of galaxies, even those in which star formation turned off a long time ago. The short burst GRB 050813 belongs to the small set of short bursts for which to date it has not been possible to precisely define the host galaxy.

\subsection{GRB 050813}

According to its observed duration ( $T_{90}$, see below), GRB 050813 can be associated with the class of short bursts with very high $(99.9 \%)$ probability (Donaghy et al. 2006). In addition, its measured spectral lag is consistent with zero, another important property of short bursts (Norris \& Bonnell 2006; Donaghy et al. 2006). Furthermore, the small original Swift XRT error circle encompasses parts of an anonymous cluster of galaxies with ellipticals inside and close to the error circle (Gladders et al. 2005; Gorosabel et al. 2005; Prochaska et al. 2006). Taken together, these observations suggest that GRB 050813 should be considered as a typical short burst.

GRB 050813 was detected by the Swift satellite on 2005 August 13, 6:45:09.76 UT (Retter et al. 2005). Its duration in the $15-350 \mathrm{keV}$ band was $0.6 \pm 0.1 \mathrm{~s}$ (Sato et al. 2005), making it the third short burst after GRBs 050509B and 050724 that Swift localized quickly and precisely. It is reminiscent of GRB 050509B, which had a very faint X-ray afterglow (Gehrels et al. 2005). Ground analysis of the $X$-ray data revealed a faint, uncataloged source at coordinates $\alpha=16^{\mathrm{h}} 07^{\mathrm{m}} 57.0^{\mathrm{s}}, \delta=+11^{\circ} 14^{\prime} 52^{\prime \prime}$ (J2000.0) with an uncertainty of $10^{\prime \prime}$ radius (Morris et al. 2005). This position was later refined by Moretti et al. (2006) to $\alpha=$ $16^{\mathrm{h}} 07^{\mathrm{m}} 57.07^{\mathrm{s}}, \delta=+11^{\circ} 14^{\prime} 54.2^{\prime \prime}(\mathrm{J} 2000.0)$ with an uncertainty of $6.5^{\prime \prime}$ radius; an even smaller error region was reported by Prochaska et al. (2006). No optical or near-infrared afterglow candidate was found. $\mathrm{Li}$ (2005) reported an unfiltered upper limit of $18.6 \mathrm{mag}$ at $49.2 \mathrm{~s}$ after the burst. UVOT observations started $102 \mathrm{~s}$ after the trigger, and a $3 \sigma$ upper limit of $V=19.1$ was derived from a $188 \mathrm{~s}$ exposure (Blustin et al. 2005). Sharapov et al. (2005) found a limiting $I$-band magnitude of $\sim 21$ at $10.52 \mathrm{hr}$ after the burst, while Bikmaev et al. (2005) reported an $R$-band upper limit of $\sim 23$ at $12.75 \mathrm{hr}$ after the event.

Spectroscopy of galaxies close to and inside the XRT error circle revealed a mean redshift of $z=0.72$ (Berger 2005; Foley et al. 2005; Prochaska et al. 2006), indicating the possibility that this may also be the redshift of the GRB. This was later refuted by Berger (2006), who argued that the host is a background galaxy at a (photometric) redshift of about 1.8 , possibly related to a background cluster of galaxies. This would make GRB 050813 the second most distant (after GRB 060121; de Ugarte Postigo et al. 2006; Levan et al. 2006) short burst for which a redshift could be estimated.

Here we report on a deep follow-up observing campaign of GRB 050813 with telescopes at Paranal, Chile, as well as at Calar Alto and at the Observatorio Sierra Nevada (OSN), Spain. The constraints we can set on any SN component following this burst, as well as the faintness of its optical afterglow, match well what is known so far about the properties of short bursts. Throughout this paper we adopt a universe model with $H_{0}=71 \mathrm{~km} \mathrm{~s}^{-1} \mathrm{Mpc}^{-1}$, $\Omega_{M}=0.27$, and $\Omega_{\Lambda}=0.73$ (Spergel et al. 2003), which for $z=$ 0.72 yields a distance modulus of $43.22 \mathrm{mag}$. The luminosity distance is $1.36 \times 10^{28} \mathrm{~cm}$, and $1^{\prime \prime}$ corresponds to $7.23 \mathrm{kpc}$. If $z=1.8$, the corresponding numbers are $45.7 \mathrm{mag}, 4.26 \times 10^{28}$ $\mathrm{cm}$, and $8.55 \mathrm{kpc}$.
TABLE 1

ObSERVING Log OF THE GRB 050813 Field

\begin{tabular}{cccccc}
\hline \hline $\begin{array}{c}\text { Date } \\
\text { (days) }\end{array}$ & $\begin{array}{c}t-t_{0}{ }^{\mathrm{a}} \\
(\text { days })\end{array}$ & $\mathrm{Mag}^{\mathrm{b}}$ & $\begin{array}{c}\text { Exposure } \\
(\mathrm{s})\end{array}$ & Filter & Telescope \\
\hline $13.8333 \ldots \ldots \ldots \ldots . .$. & 0.5519 & 22.8 & $10 \times 600$ & $I$ & $1.5 \mathrm{~m}$ OSN \\
$13.8708 \ldots \ldots \ldots \ldots$. & 0.5894 & 23.3 & $23 \times 180$ & $R$ & $2.2 \mathrm{~m}$ CAFOS \\
$14.8475 \ldots \ldots \ldots \ldots .$. & 1.5661 & 23.1 & $24 \times 300$ & $R$ & 2.2 m CAFOS \\
$19.0606 \ldots \ldots \ldots \ldots$. & 5.7792 & 25.1 & $10 \times 200$ & $I$ & 8.2 m FORS2 \\
$24.9901 \ldots \ldots \ldots \ldots$. & 11.7087 & 25.5 & $10 \times 200$ & $I$ & 8.2 m FORS2 \\
\hline
\end{tabular}

${ }^{\mathrm{a}} t_{0}=2005$ August 13.2814, the time of the burst. All dates refer to 2005 August and give the time of the start of the first exposure.

$\mathrm{b}$ The limiting magnitude of the combined image.

\section{OBSERVATIONS AND DATA REDUCTION}

A first imaging of the GRB error box was performed with the $1.5 \mathrm{~m}$ telescope at OSN and the Calar Alto $2.2 \mathrm{~m}$ telescope equipped with CAFOS starting 0.5 days after the burst (Gorosabel et al. 2005). Unfortunately, these observations resulted only in upper limits for the magnitude of any optical transient (Table 1). In order to set constraints on a rising SN component, we then carried out deep follow-up observations using VLT FORS2 in standard resolution (SR) imaging mode with a scale of $0.25^{\prime \prime}$ pixel $^{-1}$ (field of view $6.8^{\prime} \times 6.8^{\prime}$ ). Observations were performed in the Bessel $I$ band in order to minimize the potential influence of host extinction on the discovery of a fading (afterglow) or a rising (supernova) source. A first run was performed on August 19.061-19.088 UT, 5.8 days after the burst. Ten frames were obtained, each with $200 \mathrm{~s}$ exposure time each. Seeing conditions were very good, $\sim 0.5^{\prime \prime}$. A second run using the same instrumental setup was performed on August 24.990-25.017 UT, 11.7 days after the burst. Atmospheric seeing conditions were even better than during the first observing run, approaching $0.35^{\prime \prime}$. Both nights were photometric.

The FORS2 images were bias-subtracted and flat-fielded with the standard reduction procedures provided within IRAF. ${ }^{17}$ Frames obtained on the same night and in the same band were summed together in order to increase the signal-to-noise ratio. Photometry was performed with standard point-spread function (PSF) fitting using the DAOPHOT II image data analysis package PSFfitting ${ }^{18}$ algorithm (Stetson 1987) within the MIDAS platform. ${ }^{19}$ In addition, we performed aperture photometry using the IRAF aperture photometry package APPHOT.

Additional spectroscopic observations covering the entire original $r=10^{\prime \prime}$ XRT error circle (Morris et al. 2005) were performed with the VIMOS Integral Field Unit at the ESO VLT starting $20 \mathrm{hr}$ after the burst. Unfortunately, these observations could not be implemented in this study due to technical problems with the data.

Figure 1 shows the Swift XRT 90\% containment radius reported by Morris et al. (2005; large circle), the refined error circle by Moretti et al. (2006; small circle) and, as a small ellipse, the reanalyzed X-ray error box (68\% containment radius) given by Prochaska et al. (2006). In the original $r=10^{\prime \prime}$ XRT error circle we identify 11 sources, designated by the letters $\mathrm{C}, \mathrm{D}, \mathrm{E}, \mathrm{F}$, and the numbers 1-7. Note that $\mathrm{B}=\mathrm{X}, \mathrm{C}=\mathrm{B}, 4=\mathrm{B}^{*}$, and $\mathrm{E}=\mathrm{C}$ in the nomenclature of Prochaska et al. (2006). The X-ray error box published by Prochaska et al. (2006) contains only two sources, of which source 6 is the one identified by Berger (2006) as the

\footnotetext{
${ }^{17}$ See http://iraf.noao.edu.

18 The PSF-fitting photometry is accomplished by modeling a two-dimensional Gaussian profile with two free parameters (the half-width at half-maxima along $x$ and $y$-coordinates of each frame) on at least five unsaturated bright stars in each image.

${ }^{19} \mathrm{See}$ http://www.eso.org/sci/data-processing/software/esomidas.
} 


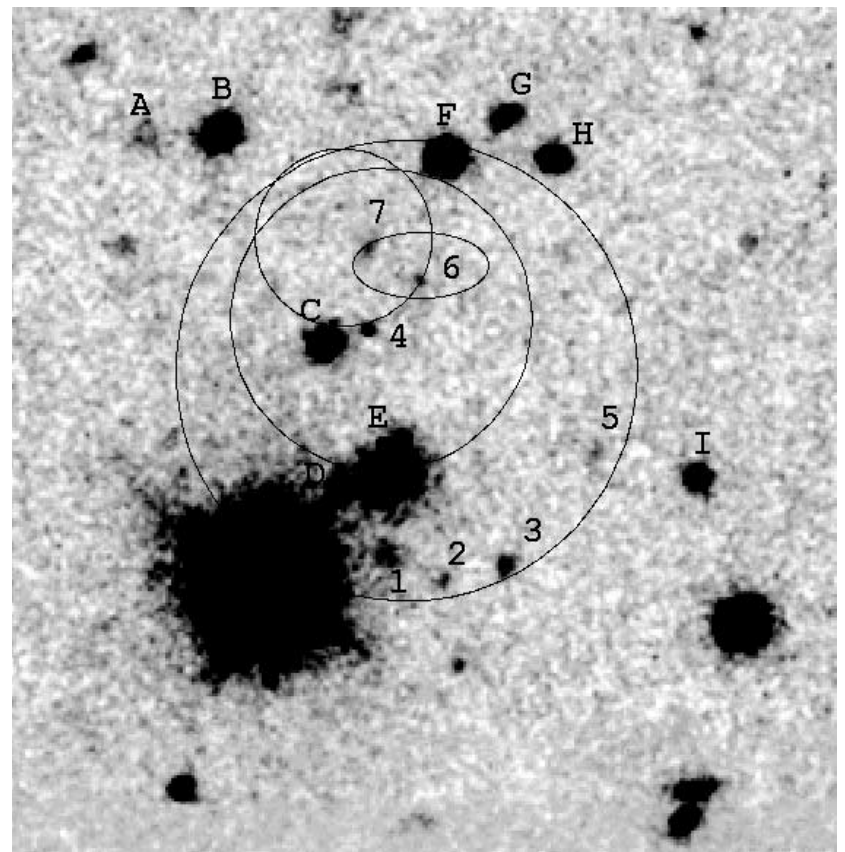

FIG. 1.-VLT I-band image of the GRB field obtained 11 days after the burst, showing the original $10^{\prime \prime}$ (radius) XRT error circle of GRB 050813 (Morris et al. 2005; large circle), the refined error circle by Moretti et al. (2006; small circle centered around source 4), the revised error ellipse (Prochaska et al. 2006), the refined error circle by Butler (2007; small circle centered around source 7), and the objects listed in Tables 2 and 3.

possible host galaxy possibly related to a cluster of galaxies (Berger 2008) at $z=1.8$. Nothing can be said at this stage about the redshift of source 7, however. Here we assume that it is a member of the cluster of galaxies at $z=0.72$ (Berger 2005; Foley et al. 2005; Prochaska et al. 2006).

\section{RESULTS}

Our two FORS2 observing runs were arranged such that they would allow us to search for a fading (afterglow), as well as for a rising (supernova), component following GRB 050813, supposing $z=0.72$. Initially we searched for a transient isolated point source in the original $10^{\prime \prime}$ XRT error circle, but we did not find one. The fact that sources 2, 5, and 6 (Fig. 1; Table 2) are not detected in the combined image of the first VLT FORS2 observing run might be due to the presence of the Moon, causing an enhanced sky background level. During the second FORS2 run the sky background was much lower and the seeing even better than during the first observing run. We conclude that any well-isolated afterglow or supernova in this field was fainter than the magnitude limits at the time of the two FORS2 observing runs, $I=25.1$ and 25.5 , respectively.

\subsection{Search for a Fading Afterglow Component}

Based on our deep FORS2 observing runs, we searched for a potential fading afterglow superimposed on the brightest extended sources (galaxies) in the field (Table 3). No evidence for variability due to an underlying transient source was found. Prochaska et al. (2006) identified objects C and E as elliptical galaxies (Fig. 1), with $\mathrm{C}$ being the most likely host candidate based on its location relative to their revised elliptical error circle. In our images source E appears to have an irregular halo, which does not support its classification as an elliptical. Image subtraction did not reveal any transient source superimposed on this galaxy.
TABLE 2

The Photometry of the Fainter Sources in the XRT Error Circle

\begin{tabular}{ccccc}
\hline \hline No. $^{a}$ & R.A. & Decl. $^{\mathrm{b}}$ & $I$ Run $1^{\mathrm{c}}$ & $I$ Run $2^{\mathrm{c}}$ \\
\hline $1 \ldots \ldots \ldots$. & 160757.00 & +111443.83 & $24.7<I<24.9$ & $24.4<I<25.4$ \\
$2 \ldots \ldots \ldots$. & 160756.85 & +111442.91 & $>25.1$ & $24.4<I<25.5$ \\
$3 \ldots \ldots \ldots$. & 160756.66 & +111443.58 & $24.69 \pm 0.24$ & $24.44 \pm 0.10$ \\
$4 \ldots \ldots \ldots$. & 160757.07 & +111453.65 & $24.63 \pm 0.30$ & $24.67 \pm 0.13$ \\
$5 \ldots \ldots \ldots .$. & 160756.40 & +111448.35 & $>25.1$ & $25.47 \pm 0.25$ \\
$6 \ldots \ldots \ldots$. & 160756.91 & +111455.91 & $>25.1$ & $25.64 \pm 0.28$ \\
$7 \ldots \ldots \ldots .$. & 160757.07 & +111457.43 & $24.7<I<25.1$ & $25.41 \pm 0.25$ \\
\hline
\end{tabular}

NotE.- Units of right ascension are hours, minutes, and seconds, and units of declination are degrees, arcminutes, and arcseconds.

${ }^{a}$ The numbering follows Fig. 1.

${ }^{\mathrm{b}}$ Epoch J2000.0.

${ }^{c}$ Run 1 and run 2 refer to the first and second VLT FORS observations, respectively.

In order to obtain an upper limit on the possible detection of an afterglow (or a SN) in the first (second) epoch FORS2 image superimposed source E, we artificially added point sources of different magnitudes to $\mathrm{E}$ and then performed aperture photometry. These point sources were selected from the second epoch image. All pixels of the second epoch image were then set to zero except the pixels of the selected point source of known magnitude, and the resulting image was then shifted and added to the first-epoch image. This analysis showed that we would have been able to detect (at $3 \sigma$ ) a fading afterglow superimposed on this galaxy if its $I$-band magnitude had been 23.5 at the time of the first FORS2 observation.

\subsection{Upper Limits on a Rising Supernova Component}

One of the main observational characteristics of a short burst should be the absence of a SN component in the late-time afterglow (Hjorth et al. 2005a), as the merger is not expected to result in the kind of radioactivity-powered optical display typical for thermonuclear (Type Ia) and core-collapse (Types II and Ib/c) supernovae. However, mergers may have subrelativistic explosions with low amounts of ejected mass (Li \& Paczyński 1998; Kulkarni 2005), but they should have small luminosities. In agreement with these expectations, strong upper limits could be set so far on any potential SN component accompanying short bursts (cf. Hjorth et al. 2005a; Fox et al. 2005).

The constraints we can place on a rising SN component for GRB 050813 are less severe, given the potentially relatively high redshift of this burst. For the cosmological parameters employed

TABLE 3

The Objects Used for the Calibration of the Photometry (A, B, F, G, H, I) and the Brightest GalaXies in the XRT Error Circle (C, D, E)

\begin{tabular}{|c|c|c|c|}
\hline No. ${ }^{a}$ & R.A. ${ }^{b}$ & Decl. $^{\mathrm{b}}$ & $I$ \\
\hline А & 160757.72 & +111502.24 & $24.68 \pm 0.35$ \\
\hline 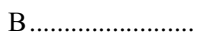 & 160757.50 & +111502.13 & $21.83 \pm 0.09$ \\
\hline 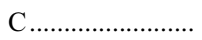 & 160757.19 & +111453.15 & $22.43 \pm 0.12$ \\
\hline 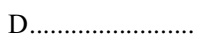 & 160757.16 & +111446.86 & $23.38 \pm 0.22$ \\
\hline 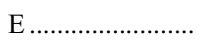 & 160757.01 & +111447.61 & $22.74 \pm 0.28$ \\
\hline F & 160756.85 & +111501.80 & $20.88 \pm 0.03$ \\
\hline 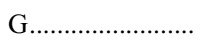 & 160756.66 & $+11 \quad 1502.87$ & $23.61 \pm 0.19$ \\
\hline 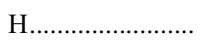 & 160756.53 & +111501.11 & $22.85 \pm 0.14$ \\
\hline I & 160756.10 & +111447.34 & $23.50 \pm 0.17$ \\
\hline
\end{tabular}

Note.-Units of right ascension are hours, minutes, and seconds, and units of declination are degrees, arcminutes, and arcseconds.

a The numbering follows Fig. 1.

b Epoch J2000.0. 
here, SN 1998bw (Galama et al. 1998) redshifted to $z=0.72$ would have magnitudes of $I=24.7$ and 23.9 during our first and second VLT FORS observing run, respectively, after taking into account a Galactic reddening of $E(B-V)=0.056 \mathrm{mag}$ (Schlegel et al. 1998) in the direction of GRB 050813. At that brightness level we would have detected the SN if it were not superimposed on a much brighter host or strongly extinguished by dust. More precisely, we conclude that at the time of our second FORS2 observation any supernova following GRB 050813 would have been at least about $1.5 \mathrm{mag}$ less luminous than SN 1998bw. While constraints placed on any $\mathrm{SN}$ component underlying the afterglow of, e.g., GRB 050509B (Hjorth et al. 2005a) and GRB 050709 (Fox et al. 2005; Covino et al. 2006) are much stronger, this makes a potential SN component following GRB 050813 already fainter than any of the 11 GRB SNe of long bursts known to date (Ferrero et al. 2006, their Fig. 6).

On the other hand, we would have been able to detect (at $3 \sigma$ ) a rising SN component superimposed on the bright galaxy E (Fig. 1) only if its $I$-band magnitude had been 23.5 at the time of the second FORS2 observation. In other words, a SN 1998bw-like component would be missed in this case. The same holds for a typical Type Ia supernova (Krisciunas et al. 2003), which would have had $I=26.9$ and 25.4 at the time of our first and second FORS2 observing run, respectively.

\section{DISCUSSION}

Short bursts, by phenomenological classification introduced by Kouveliotou et al. (1993), are bursts whose $T_{90}$ duration measured with BATSE is less than $2 \mathrm{~s}$. Even though it was already known in the 1990s that $T_{90}$ is a function of energy (and of detector properties), this definition, because of its simplicity, has been widely used even in the HETE-2 and the Swift eras. In principle, having now much more observational data at hand for individual bursts than in the BATSE era, this phenomenological definition/classification scheme calls for a more accurate, namely, physical, classification scheme.

It is clear that the classification of individual bursts with respect to the nature of their progenitor is difficult. Recent investigations have tackled this problem and have led to the suggestion of much more than just one criterion in order to classify a GRB (Donaghy et al. 2006; Norris \& Bonnell 2006). As long as no consensus has been reached in the literature on what the ultimate criteria are for a burst to be classified as being due to a merger event, in several cases only arguments that favor one scenario over the other (merger vs. collapse) can be provided. The detection or nondetection of a SN signal plays a key role in this approach but has come into question recently (see Gehrels et al. 2006; Fynbo et al. 2006; Della Valle et al. 2006; Gal-Yam et al. 2006; Zhang 2006). This leaves the nature of the host galaxy as the strongest argument to detect a GRB due to a merger event, namely, if the host is an elliptical galaxy. But the potentially broad range in merger times and hence distances of the merger events from their host galaxies (cf. Belczynski et al. 2006) might also call into question the application of this criterion. GRB 050813 belongs to those bursts that demonstrate all these problems in detail.

One of the main goals of our observing runs was the localization of the afterglow, and hence the identification of the GRB host galaxy. Basically, the host cannot be identified with certainty, and we have to consider other arguments that favor or disfavor any galaxy visible on the deep FORS2 $I$-band images of the XRT error circle as the potential host. GRB 050813 then joins the increasing list of short bursts with no detected optical afterglow, starting with GRB 050509B (Bloom et al. 2006; Castro-Tirado et al. 2005; Gehrels et al. 2005; Hjorth et al. 2005a). Using the

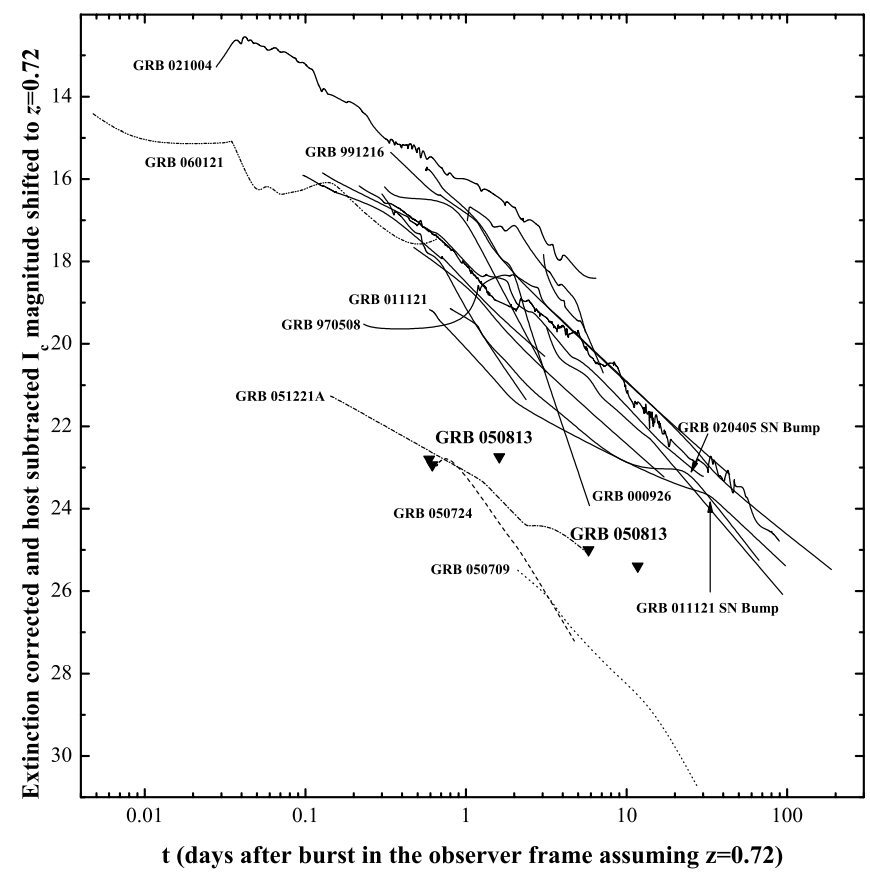

FIG. 2.- The $I$-band light curves of all afterglows from the Golden Sample of Kann et al. (2006) after correction for Galactic and host extinction and after shifting them to a common redshift of $z=0.72$, the potential redshift of GRB 050813. Two long GRB supernova rebrightenings are indicated. Also shown are the $I$-band afterglows of the short bursts GRB 050709, 050724, 051221A, and 060121 shifted in a similar way, and our upper limits on any afterglow or supernova from GRB 050813 (upside-down triangles). For GRB 060121 a redshift of $z=4.6$ (de Ugarte Postigo et al. 2006) is assumed here.

upper limits on the afterglow of GRB 050813 (Table 1), we can follow Kann et al. (2006) and place the properties of this afterglow in the context of other known GRB afterglows (Fig. 2). The long-burst afterglows shown in Figure 2 by solid lines are those from the "Golden Sample" of Kann et al. (2006), i.e., those that have sufficient $I$-band data. In addition, we analyzed the available afterglow data on the short bursts GRB 050709 (Hjorth et al. 2005b; Fox et al. 2005; Covino et al. 2007), GRB 050724 (Berger et al. 2005; Malesani et al. 2007), GRB 051221A (Soderberg et al. 2006), and GRB 060121 (Levan et al. 2006; de Ugarte Postigo et al. 2006) in an analogous way and also included them in Figure 2 (see the Appendix for details). As can be seen, short-burst optical afterglows are intrinsically very faint, with the afterglows of GRB 050724 and GRB 051221A being about 3 mag fainter than any long-burst afterglow in the sample, and GRB 050709 being 4 mag fainter at 1 day after the burst and assuming $z=0.72$ (in agreement with the predictions for short-burst afterglows; Panaitescu et al. 2001). They are also significantly fainter than intrinsically faint afterglows of some long GRBs, such as GRB 021211. Only the afterglow of GRB 060121 is comparable with the typical afterglows of long GRBs. The upper limits on the optical afterglow of GRB 050813 show that its luminosity was also far below typical luminosities of (extinction-corrected) afterglows of long bursts. On the other hand, it matches the luminosity region occupied so far by the afterglows of the short bursts (with GRB 060121 being the only exception).

Figure 1 shows that there are only two sources in the XRT error ellipse (Prochaska et al. 2006), while there are at least three additional sources in the refined error circle (Moretti et al. 2006). The former might favor a burst related to the very faint sources 6 and 7 (source 6 appears pointlike in our images), but it does not even exclude an event in the outer halo of source $\mathrm{C}$, an elliptical 


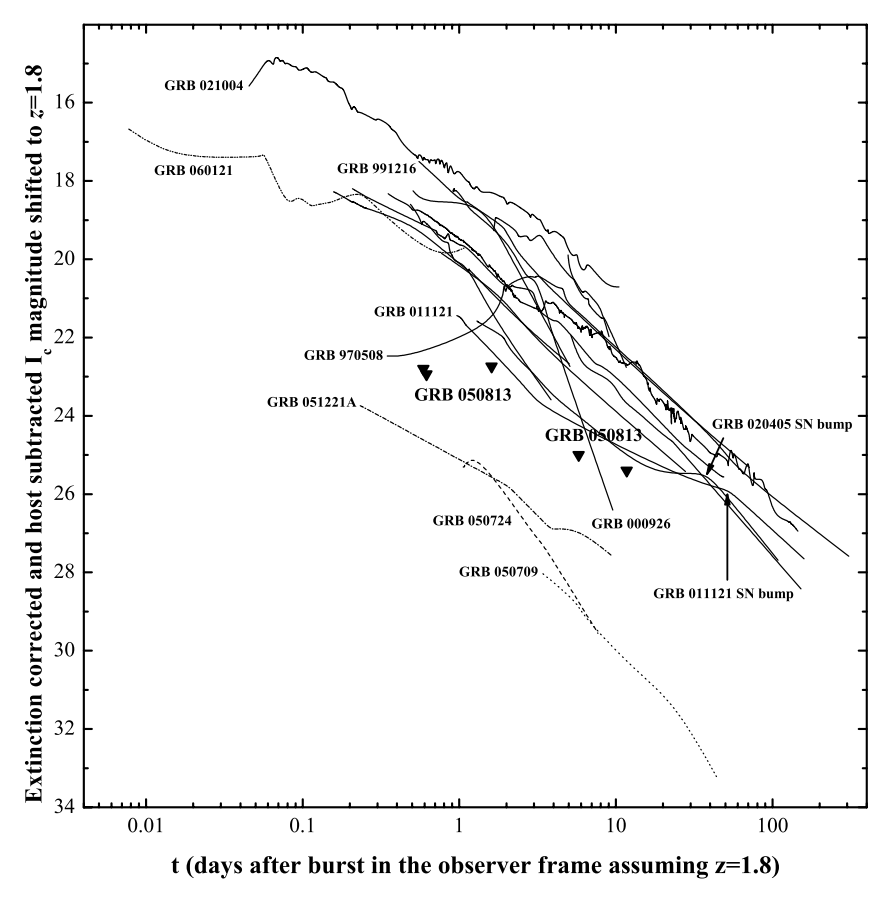

FIG. 3.- Same as Fig. 2, but for a redshift of 1.8.

galaxy at a redshift of 0.719 (Prochaska et al. 2006). The minimum distance between the border of the error ellipse and the center of this galaxy is $3.2^{\prime \prime}$, corresponding to a projected distance of $23 \mathrm{kpc}$. This is less than the projected distance of the error circle of GRB 050509B from the center of its suspected host, an elliptical galaxy at a redshift of $z=0.225$ (Gehrels et al. 2005). In addition, the minimum angular distance between source $\mathrm{E}$ and the border of the error ellipse is 7.1", corresponding to a projected distance of $51 \mathrm{kpc}$. Even this is within the range predicted by recent models of merging compact objects (see Belczynski et al. 2002; Perna \& Belczynski 2002). The error circle determined by Moretti et al. (2006) is much larger, and thus allows not only source $\mathrm{C}$ but also galaxy E at $z=0.73 \pm 0.01$ (Prochaska et al. 2006) to be the potential host of GRB 050813. This galaxy was classified by Prochaska et al. (2006) as an elliptical galaxy, while our images show morphology that points either to a spiral or to an irregular galaxy. The nature of the fifth, pointlike source in the refined error circle, source 4 , remains undetermined.

While this paper was submitted, a new revised XRT error circle was reported by Butler (2007). This revised error circle is $3.8^{\prime \prime}$ in radius and centered close to a faint edge-on galaxy. This galaxy (source 7; see Fig. 1) was only marginally detected during the first FORS observations. A comparison with the second FORS observations 6 days later does not provide convincing evidence for a photometric variability due to an underlying point source.

To summarize, our optical data do not reveal either an afterglow or a SN component. If GRB 050813 was occurring in a cluster of galaxies at a redshift of $z=0.72$, as might be indicated by the surrounding galaxy population, then its projected distance from its potential host galaxy could have been of the order of less than 4 to some dozen kpc, depending on the chosen potential host galaxy. The nondetection of the afterglow is well in accord with the faintness of optical afterglows following short bursts (Fig. 2). On the other hand, if the burster had been at $z=1.8$ (Berger 2006), no SN 1998bw-like component would have been detectable in our images, and any afterglow component would have been correspondingly fainter than in the former case (Fig. 3). But even in this case the upper limits we can set on any optical after- glow are consistent with the hypothesis that GRB 050813 was a typical member of the short bursts.

We thank the staff at ESO/Paranal, in particular C. Dumas, P. D. Lynam, P. Gandhi, N. Huélamo, and E. Jehin, for performing the observations and for additional efforts related to that. We thank the CAHA and OSN staff for excellent support during some of the observations presented here. P. F., D. A. K., and S. K. acknowledge financial support from DFG grant K1 766/13-2 and from the German Academic Exchange Service (DAAD) under grant $\mathrm{D} / 05 / 54048$. The research activity of J. G. is supported by the Spanish Ministry of Science through projects AYA 200401515 and ESP 2005-07714-C03-03. We thank the second referee for a rapid reply and a constructive report.

\section{APPENDIX}

\section{THE LIGHT CURVES OF THE SHORT-BURST AFTERGLOWS}

In Figure 2 we included those four GRBs that have both an optical afterglow and a redshift derived from either host galaxy spectroscopy or photometry (GRB 060121; de Ugarte Postigo et al. 2006) up to 2006 October.

We take data from the following works: GRB 050709 (Hjorth et al. 2005b; Fox et al. 2005; Covino et al. 2006), GRB 050724 (Berger et al. 2005; Malesani et al. 2007), GRB 051221A (Soderberg et al. 2006), and GRB 060121 (Levan et al. 2006) de Ugarte Postigo et al. 2006).

For GRB 050709, we derive a decay slope of $\alpha=1.68 \pm$ 0.15 from the $R_{\mathrm{C}}$-band light curve. Fox et al. (2005) noted that the late Hubble Space Telescope (HST) data indicate a steepening of the light-curve decay, possibly due to a jet break. Using the $R_{\mathrm{C}}$-band decay index, we find a rebrightening (significant at the $5 \sigma$ level) in the $H S T$ data, but only marginal evidence that the afterglow is fainter than expected from the early decay in the last HST detection. This result is in accordance with Watson et al. (2006). The light curve shown in Figure 2 is composed of the $R_{\mathrm{C}}$ data shifted to the $H S T \mathrm{~F} 814 \mathrm{~W}$ zero point, plus the $H S T$ data. From the $V, R_{\mathrm{C}}, F 8, K^{\prime}$ spectral energy distribution (SED), we derive a steep uncorrected spectral slope $\beta_{0}=1.71 \pm 0.17$. This is indicative of additional source-frame extinction. As the host is a blue dwarf galaxy (Fox et al. 2005), we assumed SMCtype dust (Pei 1992). A free fit implies $\beta=0.26 \pm 1.16$ and a host extinction of $A_{V}$ (host) $=1.46 \pm 1.07 \mathrm{mag}$, a very high value indeed. As the single $K^{\prime}$ data point has a very large error $(0.7 \mathrm{mag})$, this value may not be trustworthy. For a progenitor that has traveled far from its birthplace, an unstratified surrounding medium is expected (density $\rho \propto r^{0}$ ). We fixed $\beta$ to the value derived from the prebreak decay slope $\alpha_{1}$, and find $\beta=1.12$ and $A_{V}$ (host) $=0.67 \pm 0.19 \mathrm{mag}$. We used these parameters to correct and shift the light curve.

For GRB 050724, the Galactic extinction is high and not well determined. We follow Malesani et al. (2007), who argue, based on the X-ray-to-optical SED, for $E_{B-V}=0.49$. After correcting for this extinction, we find $\beta=0.76 \pm 0.07$ and no evidence for source-frame extinction, in accordance with Malesani et al. (2007). The light curve is mostly $I_{\mathrm{C}}$ data anyway; we add $V, R_{\mathrm{C}}$, and $K$ data shifted to the $I_{\mathrm{C}}$ zero point.

In the case of GRB $051221 \mathrm{~A}$, we find that the light curve decays as a single power law with a slope $\alpha=0.94 \pm 0.03$, in accordance with Soderberg et al. (2006). We derive a flat spectral 
slope $(\beta=-0.16 \pm 0.84)$ from the $r^{\prime} i^{\prime} z^{\prime}$ SED, but caution that the errors of the $i^{\prime}$ and $z^{\prime}$ data are very large. Assuming an unstratified surrounding medium and a cooling frequency blueward of the optical bands, we derive $\beta=0.62$ (coupled with a typical power-law index of the electron distribution function of $p=2.25$; cf. Kann et al. 2006). We used this spectral slope and assume no additional extinction to shift the light curve.

Combining the data from Levan et al. (2006) and de Ugarte Postigo et al. (2006) of GRB 060121, we find that the zero points of the two data sets differ. We shifted the data from de Ugarte Postigo et al. (2006) to the fainter zero point of Levan et al. (2006). The light curve has a complex shape and seems to include several rebrightenings (Fig. 2). It is composed of $I_{\mathrm{C}}$ data and $R_{\mathrm{C}}$ data shifted to the $I_{\mathrm{C}}$ zero point. We used the redshift and host galaxy extinction derived by de Ugarte Postigo et al. (2006), assuming the more probable redshift of $z=4.6$ and a spectral slope in the optical of $\beta=0.6$, as derived by the authors cited above.

In all cases, except for GRB 060121, the afterglow data do not contain any host contribution. For GRB 060121, we used a host galaxy magnitude derived from the HST measurements (Levan et al. 2006). To correct for Galactic extinction, we used the value derived from the maps of Schlegel et al. (1998) for GRB 050709, 051221A, and 060121, and $E_{B-V}=0.49 \mathrm{mag}$ for GRB 050724 (as suggested by Malesani et al. 2007).
Aloy, M. A., Janka, H.-T., \& Müller, E. 2005, A\&A, 436, 273

Belczynski, K., Bulik, T., \& Kalogera, V. 2002, ApJ, 571, L147

Belczynski, K., et al. 2006, ApJ, 648, 1110

Berger, E. 2005, GCN Circ. 3801, http://gen.gsfc.nasa.gov/gen3/3801.gcn3 . 2006, in AIP Conf. Proc. 836, Gamma-Ray Bursts in the Swift Era, ed.

S. S. Holt, N. Gehrels, \& J. A. Nousek (New York: AIP), 33

. 2008, in Swift and GRBs: Unveiling the Relativistic Universe (Bologna: Italian Physical Soc.), in press

Berger, E., et al. 2005, Nature, 438, 988

Bikmaev, I., et al. 2005, GCN Circ. 3797, http://gcn.gsfc.nasa.gov/gcn3/3797.gcn3

Bloom, J. S., Dupree, A., Chen, H-W., \& Prochaska, J. X. 2005, GCN 3672, http://gcn.gsfc.nasa.gov/gcn3/3672.gcn3

Bloom, J. S., et al. 2006, ApJ, 638, 354 $2007, \mathrm{ApJ}, 654,878$

Blustin, A. J., et al. 2005, GCN Circ. 3791, http://gen.gsfc.nasa.gov/gen3/3791 .gen 3

Butler, N. R. 2007, AJ, 133, 1027

Castro-Tirado, A. J., et al. 2005, A\&A, 439, L15

Covino, S., et al. 2006, A\&A, 447, L5

Della Valle, M., et al. 2006, Nature, 444, 1050

de Ugarte Postigo, A., et al. 2006, ApJ, 648, L83

Donaghy, T. Q., et al. 2007, ApJ, submitted (astro-ph/0605570)

Faber, J. A., Baumgarte, T. W., Shapiro, S. L., \& Taniguchi, K. 2006, ApJ, 641, L93

Ferrero, P., et al. 2006, A\&A, 457, 857

Foley, R. J., Bloom, J. S., \& Chen, H.-W. 2005, GCN Circ. 3808, http://gen.gsfc nasa.gov/gen $3 / 3808$.gcn 3

Fox, D. B., et al. 2005, Nature, 437, 845

Frederiks, D. D., et al. 2007, Astron. Lett., 33, 19

Fryer, C. L., Woosley, S. E., \& Hartmann, D. H. 1999, ApJ, 526, 152

Fynbo, J. P. U., et al. 2006, Nature, 444, 1047

Galama, T. J., et al. 1998, Nature, 395, 670

Gal-Yam, A., et al. 2006, Nature, 444, 1053

Gehrels, N., et al. 2005, Nature, 437, 851 2006, Nature, 444, 1044

Gladders, M., Berger, E., Morell, N., \& Roth, M. 2005, GCN Circ. 3798, http://gen gsfc.nasa.gov/gen3/3798.gcn3

Golenetskii, S., et al. 2005, GCN Circ. 4197, http://gcn.gsfc.nasa.gov/gen3/ 4197.gcn3

Gorosabel, J., et al. 2005, GCN Circ. 3796, http:/gcn.gsfc.nasa.gov/gcn3/3796 .gcn 3

2006, A\&A, 450, 87

Hjorth, J., et al. 2003, Nature, 423, 847

2005a, ApJ, 630, L117

2005b, Nature, 437, 859

\section{REFERENCES}

Kann, D. A., Klose, S., \& Zeh, A. 2006, ApJ, 641, 993

Kouveliotou, C., et al. 1993, ApJ, 413, L101

Krisciunas, K., et al. 2003, AJ, 125, 166

Kulkarni, S. R. 2005, preprint (astro-ph/0510256)

La Parola, V., et al. 2006, A\&A, 454, 753

Levan, A. J., et al. 2006, ApJ, 648, L9

Li, L. X., \& Paczyński, B. 1998, ApJ, 507, L59

Li, W. 2005, GCN Circ. 3794, http://gcn.gsfc.nasa.gov/gen3/3794.gcn3

Malesani, D., et al. 2006, GCN Circ. 4561, http:/gcn.gsfc.nasa.gov/gcn3/4561 gcn3

. 2007, A\&A, 473, 77

Moretti, A., et al. 2006, A\&A, 448, L9

Morris, D. C., et al. 2005, GCN Circ. 3790, http://gcn.gsfc.nasa.gov/gcn3/3790 gen 3

Norris, J. P., \& Bonnell, J. T. 2006, ApJ, 643, 266

Oechslin, R., \& Janka, H.-Th. 2006, MNRAS, 368, 1489

Ofek, E. O., et al. 2006, ApJ, 652, 507

Paczyński, B. 1998, ApJ, 494, L45

Panaitescu, A., Kumar, P., \& Narayan, R. 2001, ApJ, 561, L171

Pedersen, K., et al. 2005, ApJ, 634, L17

Pei, Y. C. 1992, ApJ, 395, 130

Perna, R., \& Belczynski, K. 2002, ApJ, 570, 252

Pian, E., et al. 2006, Nature, 442, 1011

Prochaska, J. X., Chen, H.-W., Bloom, J. S., \& Stephens, A. 2005, GCN Circ. 3679, http://gcn.gsfc.nasa.gov/gen3/3679.gcn3

Prochaska, J. X., et al. 2006, ApJ, 642, 989

Racusin, J. L., et al. 2006, GCN Circ. 5378, http://gen.gsfc.nasa.gov/gcn3/5378 .gcn3

Retter, A., et al. 2005, GCN Circ. 3788, http://gcn.gsfc.nasa.gov/gen3/3788.gen3

Roming, P. W. A., et al. 2006, ApJ, 651, 985

Rosswog, S. 2005, ApJ, 634, 1202

Sato, G., et al. 2005, GCN Circ. 3793, http://gcn.gsfc.nasa.gov/gcn3/3793.gcn3

Schlegel, D. J., Finkbeiner, D. P., \& Davis, M. 1998, ApJ, 500, 525

Sharapov, D., Ibrahimov, M., Pozanenko, A., \& Rumyantsev, V. 2005, GCN Circ. 3857, http://gcn.gsfc.nasa.gov/gcn3/3857.gcn3

Soderberg, A. M., et al. 2006, ApJ, 650, 261

Spergel, D. N., et al. 2003, ApJS, 148, 175

Stanek, K. Z., et al. 2003, ApJ, 591, L17

Stetson, P. B. 1987, PASP, 99, 191

Tanvir, N. R., Chapman, R., Levan, A. J., \& Priddey, R. S. 2005, Nature, 438, 991

Watson, D., et al. 2006, A\&A, 454, L123

Woosley, S. E., \& Bloom, J. S. 2006, ARA\&A, 44, 507

Zhang, B. 2006, Nature, 444, 1010 